\title{
THE UNIVERSALITY OF FORMAL POWER SERIES FIELDS*
}

\author{
SAUNDERS MACLANE
}

In a recent paper, $\dagger$ André Gleyzal has constructed ordered fields consisting of certain "transfinite real numbers" and has established the interesting result that any ordered field can be considered as a subfield of one of these transfinite fields. These fields prove to be identical with fields of formal power series in which the exponents are allowed to range over a suitable ordered abelian group. Such fields were first introduced by Hahn, $\ddagger$ while they have been analyzed in terms of generalized valuations by Krull. $\$$

Gleyzal applied his construction of transfinite real numbers not only to the case when the coefficient field consisted of real numbers, but also to suitable fields of characteristic $p$. He conjectured that this construction should yield a "universal" field of characteristic $p$. We show here that Krull's technique can be used to establish Gleyzal's conjecture.

1. Formal power series. If $K$ is any field and $\Gamma$ any ordered abelian group (its order may be non-archimedean), we form all power series $x=\sum a_{\alpha} t^{\alpha}$ with coefficients $a_{\alpha}$ in $K$, exponents $\alpha$ in $\Gamma$, and summed over a subset $N$ of elements $\alpha$ from $\Gamma$ which is normally ordered by the given linear order in $\Gamma$. Such a series could also be written as

$$
x=a_{\alpha_{1}} t^{\alpha_{1}}+a_{\alpha_{2}} t^{\alpha_{2}}+\cdots+a_{\alpha_{\rho}} t^{\alpha_{\rho}}+\cdots,
$$

summed over all ordinal numbers $\rho$ less than a fixed $\sigma$, and with exponents $\alpha_{1}<\alpha_{2}<\ldots<\alpha_{\rho}<\ldots$ increasing monotonically. The product of two formal powers $t^{\alpha}$ and $t^{\beta}$ is defined as $t^{\alpha+\beta}$, where $\alpha+\beta$ is the sum in the group $\Gamma$. On this basis, the usual formal definitions of multiplication and addition make the set of all series (1) a field, which we denote by $K\left\{t^{\Gamma}\right\}$.

Hahn also gave a similar construction for a non-archimedean ordered group from a given ordered group $G$ (say an additive group of

* Presented to the Society, April 8, 1939.

$\dagger$ A. Gleyzal, Transfinite numbers, Proceedings of the National Academy of Sciences, vol. 23 (1937), pp. 581-587.

$\ddagger$ H. Hahn, Über die nichtarchimedischen Grössensysteme, Sitzungsberichte der Kaiserlichen Akademie der Wissenschaften, Vienna, section IIa, vol. 116 (1907), pp. 601-653.

$\S \mathrm{W}$. Krull, Allgemeine Bewertungstheorie, Journal für die reine und angewandte Mathematik, vol. 167 (1931), pp. 160-196. 
real numbers) and any ordered set $B$. One simply chooses for each $b$ in $B$ a "basis" element $e_{b}$, and then forms all sums $\alpha=\sum g_{b} e_{b}$, with $g_{b}$ in $G$, taken over the indices $b$ of a subset $M$ of $B$ which is well ordered by the given order of $B$. The formal sum of two such expressions $\alpha$ is again a similar expression. Under the usual lexicographic ordering, all these expressions constitute an ordered group $G\left\{e_{B}\right\}$.

In particular, one may start with the ordered (additive) group $R$ of all real numbers, construct the group $\Gamma=R\left\{e_{B}\right\}$ and thence the field $K=R^{\prime}\left\{t^{\Gamma}\right\}$, where $R^{\prime}$ is the real number field. This particular power series field is isomorphic to Gleyzal's field of "transfinite real numbers* with base order $B$."

Hahn also showed how the group $\Gamma$ could be redistilled from the ordered field $K=R^{\prime}\left\{t^{\Gamma}\right\}$ by considering each element in $\Gamma$ as a set of all elements in $K$ of the same "order of magnitude." If this process is applied again, the orders of magnitude in $\Gamma$ yield the original $\dagger$ ordered set $B$.

2. Algebraic closure. A root group $\Gamma$ is one with the property that for every integer $n$ and every $\alpha$ in $\Gamma$ there is a $\gamma$ in $\Gamma$ with $n \gamma=\alpha$. The particular group $R\left\{e_{B}\right\}$ is a root group.

THEOREM 1. The power series field $K\left\{t^{\Gamma}\right\}$ is algebraically closed if the coefficient field $K$ is algebraically closed and if the ordered abelian group $\Gamma$ of exponents is a root group.

Proof. In the power series field $S=K\left\{t^{\Gamma}\right\}$ we introduce a valuation $V$ by setting $V(x)=\alpha_{1}$ if $a_{\alpha_{1}} t^{\alpha_{1}}$ is the first nonvanishing term in the power series (1) for $x$. In this valuation, $\Gamma$ is the value group and $K$ the field of residue classes. Furthermore, $\ddagger S$ is maximal with respect to this valuation, in the sense that any proper extension $S^{\prime}>S$ to which the valuation $V$ has been extended must either have a larger value group or a larger residue class field than $S$.

Suppose now that $S$ is not algebraically closed, so that $S$ has a proper finite normal extension $N$. Certainly $S$ is (algebraically) perfect, so that $N / S$ is separable. The valuation $V$ of $S$ can be extended to $N$ by the usual methods, for $S$ is (topologically) perfect $\S$ with respect to $V$. The ordinary Newton polygon construction shows that

* To establish the isomorphism, observe that Gleyzal's product forms $\Pi_{\mu}$ form a multiplicative group isomorphic to $R\left\{e_{B}\right\}$, and that his transfinite real numbers or sum forms are manipulated exactly as are the formal series (1).

$\dagger$ This twofold order of magnitude process yields essentially Gleyzal's base order for $K$.

$\ddagger$ Krull, loc. cit., Theorem 26.

$\S$ Krull, loc. cit., $\$ 9$ and Theorem 27. 
each element $c$ of $N$ has a value of the form $\alpha / n$, with $\alpha$ in $\Gamma$. Since $\Gamma$ is a root group, $\alpha / n \varepsilon \Gamma$, and $\Gamma$ is thus the value group of $N$. On the other hand, the residue class field of $N$ must be an algebraic extension of the algebraically closed residue class field $K$ of $S$. Thus $N$ presents a proper extension of $S$ in which neither value group nor residue class field is extended, contrary to the maximal property of $S$.

3. Universal fields. We call a field $F$ universal if every other field $F^{\prime}$ which has the same cardinal number and the same characteristic as $F$ is isomorphic to a subfield of $F$.

THEOREM 2. A nondenumerable field $F$ is universal if and only if it contains an algebraically closed subfield $F_{0}$ which has the same cardinal number as $F$.

Proof. We need only provide a map of any $F^{\prime}$ into $F$. As in the Steinitz theory, let $T_{0}, T^{\prime}$ be respectively transcendence bases for $F_{0}$, $F^{\prime}$ over the prime subfield $P$. By the axiom of choice, the cardinal number of $T_{0}$ is some aleph. Since $P$ is at most denumerable, the usual computations with cardinal numbers then show that $P\left(T_{0}\right)$ and its algebraic extension $F_{0}$ must have the same cardinal number as does $T_{0}$. Since $F_{0}$ and $F^{\prime}$ have the same cardinal number, we conclude that $T_{0}$ and $T^{\prime}$ have the same cardinal number, so we can set $T_{0}$ and $T^{\prime}$ into one-to-one correspondence. This correspondence gives an isomorphism of $P\left(T^{\prime}\right)$ to $P\left(T_{0}\right)$. Because $F$ is algebraically closed, this isomorphism can be extended* from $P\left(T^{\prime}\right)$ to its algebraic extension $F^{\prime}$. We have proved $F_{0}$ and hence $F$ universal.

THEOREM 3. If the ordered abelian root group $\Gamma$ contains an element different from 0 , while the coefficient field $K$ is algebraically closed, then the power series field $K\left\{t^{\Gamma}\right\}$ is universal.

This field $K\left\{t^{\Gamma}\right\}$ includes all ordinary power series in $t^{\alpha}$, with $\alpha \neq 0$ in $\Gamma$, hence has at least the power of the continuum. Theorem 3 thus follows from Theorems 1 and 2. In particular, this theorem yields relatively simple universal fields whose cardinal numbers are that of the continuum; for example, the fields $K\left\{t^{\Gamma}\right\}$ with $K$ the absolutely algebraic algebraically closed field of any characteristic and $\Gamma$ the group of all rational numbers. This theorem also includes Gleyzal's conjectured fields, where $K$ is absolutely algebraic and $\Gamma$ is any one of the groups $R\left\{e_{B}\right\}$ of $\S 1$.

\section{HARVARD UNIVERSITY}

* B. L. van der Waerden, Moderne Algebra, $\$ 60$. 